TRANSACTIONS OF THE

AMERICAN MATHEMATICAL SOCIETY

Volume 358 , Number 8 , Pages 3571-3589

S 0002-9947(05)03848-1

Article electronically published on December 20, 2005

\title{
EXTENDED DEGREE FUNCTIONS AND MONOMIAL MODULES
}

\author{
UWE NAGEL AND TIM RÖMER
}

\begin{abstract}
The arithmetic degree, the smallest extended degree, and the homological degree are invariants that have been proposed as alternatives of the degree of a module if this module is not Cohen-Macaulay. We compare these degree functions and study their behavior when passing to the generic initial or the lexicographic submodule. This leads to various bounds and to counterexamples to a conjecture of Gunston and Vasconcelos, respectively. Particular attention is given to the class of sequentially Cohen-Macaulay modules. The results in this case lead to an algorithm that computes the smallest extended degree.
\end{abstract}

\section{INTRODUCTION}

Let $M$ be a finitely generated graded module over the polynomial ring $S$. If $M$ is Cohen-Macaulay, then several invariants of $M$ can be bounded using the degree of $M$. This is no longer true if $M$ is not Cohen-Macaulay. In this case, one tries to replace the degree of $M$ by an invariant that better captures the structure of $M$. One such invariant is the arithmetic degree (cf. [1]) of $M$,

$$
\operatorname{adeg} M=\sum l\left(H_{\mathfrak{p}}^{0}\left(M_{\mathfrak{p}}\right)\right) \cdot \operatorname{deg} S / \mathfrak{p},
$$

where the sum runs over the associated prime ideals of $M$.

More recently, Vasconcelos [25] has axiomatically introduced the so-called extended degrees (cf. Section 2). They are designed to provide measures for the size and the complexity of the structure of $M$. The first concrete example of an extended degree is Vasconcelos' homological degree [25]. It is recursively defined by

$$
\operatorname{hdeg} M=\operatorname{deg} M+\sum_{i=0}^{d-1}\left(\begin{array}{c}
d-1 \\
i
\end{array}\right) \operatorname{hdeg} \operatorname{Ext}_{S}^{n-i}(M, S)
$$

where $d:=\operatorname{dim} M$. Gunston ([10, cf. also [16, Lemma 4.2] has shown that among all extended degrees there is a minimal one which we just call the smallest extended degree sdeg $M$. In this paper we compare these three degrees and study their behavior when we replace $M$ by a related monomial module. This leads to various bounds.

Received by the editors June 21, 2004 and, in revised form, August 8, 2004.

2000 Mathematics Subject Classification. Primary 13D40, 13C99; Secondary 13P10, 13H10.

Key words and phrases. Extended degree functions, Buchsbaum module, sequentially CohenMacaulay module, generic initial module, lexicographic module, bounds for degree functions.

The first author gratefully acknowledges partial support by a Special Faculty Research Fellowship from the University of Kentucky.

(C)2005 American Mathematical Society Reverts to public domain 28 years from publication 
A difficulty when dealing with the smallest extended degree is that, in general, there is no formula to compute it. However, we show that such a formula does exist if $M$ is either a sequentially Cohen-Macaulay (cf. Section 3) or a Buchsbaum module (cf. Section 4). As a first application of these formulas, we show in Section 5 that every module $M$ satisfies

$$
\operatorname{deg} M \leq \operatorname{adeg} M \leq \operatorname{sdeg} M \leq \operatorname{hdeg} M .
$$

This refines Vasconcelos' Proposition 9.4.2 in [24]. Moreover, our formulas show that

$$
\operatorname{adeg} M=\operatorname{sdeg} M
$$

if $M$ is sequentially Cohen-Macaulay, and that

$$
\operatorname{sdeg} M=\operatorname{hdeg} M
$$

if $M$ is a Buchsbaum module.

The case of sequentially Cohen-Macaulay modules is of particular importance because such modules naturally occur. Indeed, write $M=F / U$ where $F$ is a free $S$-module and $U \subset F$ is a graded submodule. By now it is a standard technique to draw conclusions about $F / U$ by considering $F / \operatorname{gin}(U)$ where $\operatorname{gin}(U)$ is the generic initial module of $U$ with respect to the reverse lexicographic order on $F$ (cf. Section 2). In order to get bounds for invariants on $M$ that depend on its Hilbert function, it is often useful to compare $M=F / U$ with $F / U^{\text {lex }}$ where $U^{\text {lex }}$ is the lexicographic submodule of $F$ that has the same Hilbert function as $U$ (cf. Section 2). Both, $\operatorname{gin}(U)$ and $U^{l e x}$ are Borel-fixed (cf. Section 2), thus they are sequentially CohenMacaulay (cf. Lemma 3.4). Hence, our formulas apply and we use them to show that we have for every module $M=F / U$

$$
\operatorname{adeg} F / U \leq \operatorname{adeg} F / \operatorname{gin}(U) \leq \operatorname{adeg} F / U^{l e x}
$$

and

$$
\operatorname{sdeg} F / U=\operatorname{sdeg} F / \operatorname{gin}(U) \leq \operatorname{sdeg} F / U^{l e x} .
$$

Note that the first inequality for adeg extends a result of Sturmfels, Trung, and Vogel [23, Theorem 2.3] from ideals to submodules, whereas the equality for sdeg is due to [10 (cf. also [16]). In spite of the estimates above, it is natural to conjecture (cf. [10] and [24, page 262]) that we have for every module $M=F / U$ either always the relation

$$
\text { hdeg } F / U \geq \operatorname{hdeg} F / \operatorname{gin}(U)
$$

or

$$
\operatorname{hdeg} F / U \leq \operatorname{hdeg} F / \operatorname{gin}(U) \text {. }
$$

Since it is often possible to compare invariants of $F / U$ and $F / U^{l e x}$, one might also suspect that there is always either the relation

$$
\operatorname{hdeg} F / U \geq \operatorname{hdeg} F / U^{l e x}
$$

or

$$
\text { hdeg } F / U \leq \operatorname{hdeg} F / U^{l e x} \text {. }
$$

In fact, this work began as an attempt to prove these conjectures. Somewhat surprisingly we show in Section [6 that none of the conjectured relations is always true by exhibiting suitable modules.

Our formulas and estimates for the degree functions are in terms of the degrees of certain extension modules. In the final section, we show that these degrees can very efficiently be computed in case of monomial modules of Borel-type. As a 
consequence, we get a fast algorithm for computing sdeg $F / U$ provided we know $\operatorname{gin}(U)$.

Throughout the paper we consider finitely generated graded modules over the polynomial ring $S$. However, using [5] our results for sequentially Cohen-Macaulay and Buchsbaum modules remain valid for modules over an arbitrary Noetherian local ring $(R, \mathfrak{m})$ provided monomial modules are not involved. In the latter case, the results are still true for modules over a regular local ring $(R, \mathfrak{m})$ of dimension $n$ where the maximal ideal $\mathfrak{m}$ is generated by $x_{1}, \ldots, x_{n}$.

\section{Degree functions}

In this section we introduce several degree functions of modules. We briefly recall definitions and notation used in this paper. For unexplained terminology we refer to the book of Bruns and Herzog [3].

Throughout this paper $K$ is always an infinite field and $S=K\left[x_{1}, \ldots, x_{n}\right]$ is the polynomial ring over $K$ with its standard grading where $\operatorname{deg} x_{i}=1$ for $i=1, \ldots, n$. We denote by $\mathfrak{m}=\left(x_{1}, \ldots, x_{n}\right)$ the unique graded maximal ideal of $S$. A standard graded $K$-algebra $R$ is of the form $S / I$ for a graded ideal $I \subset S$.

Usually we denote by $M$ a finitely generated graded $S$-module of dimension $d=\operatorname{dim} M$. Its $i$-th local cohomology module is denoted by $H_{\mathfrak{m}}^{i}(M)$. The Hilbert function $H_{M}$ of $M$ is defined by

$$
H_{M}: \mathbb{Z} \rightarrow \mathbb{N}, j \mapsto \operatorname{dim}_{K} M_{j}
$$

It is well known that there exists a polynomial $P_{M}$ of degree $d-1$ such that for $j \gg 0$ we have that $H_{M}(j)=P_{M}(j)$. We write

$$
P_{M}(t)=\frac{e(M)}{(d-1) !} t^{d-1}+\ldots \text { (terms of lower degree). }
$$

We define the degree $\operatorname{deg} M$ (or multiplicity of $M$ ) to be $e(M)$ if $d>0$ and $\operatorname{deg} M=$ $l(M)$ if $d=0$. Here $l(\cdot)$ denotes the length function of a module $M$. The degree of $M$ has many nice properties, especially if $M$ is a Cohen-Macaulay module (CM module for short).

There are several attempts do define degree functions for a module $M$ that coincide with the degree if $M$ is a CM module, but also have nice properties for non-CM modules. We refer to the nice book of Vasconcelos 25] for details on this subject. One such proposal is due to Bayer and Mumford who introduced in [1] the arithmetic degree that has been studied by several authors in the last decades (see, e.g., [13, 23], or [24]). Vasconcelos [24, Proposition 9.1.2] has shown that the arithmetic degree can be computed using the formula

$$
\operatorname{adeg} M=\sum_{i=0}^{n} \operatorname{deg} \operatorname{Ext}_{S}^{i}\left(\operatorname{Ext}_{S}^{i}\left(M, \omega_{S}\right), \omega_{S}\right)
$$

where $\omega_{S}=S(-n)$ is the canonical module of $S$. But there are some disadvantages. For example, if $y \in S_{1}$ is an $M$-regular element, i.e., it is a non-zero divisor of $M$ (sometimes also called a regular hyperplane section), then

$$
\operatorname{adeg} M \leq \operatorname{adeg} M / y M \text {. }
$$

But if a degree function reflects the complexity of the module, then $M / y M$ should have a smaller degree than $M$. 
In [25], Vasconcelos axiomatically defined the following concept. A numerical function Deg that assigns to every finitely generated graded $S$-module a nonnegative integer is said to be an extended degree function if it satisfies the following conditions:

(i) If $L=H_{\mathfrak{m}}^{0}(M)$, then $\operatorname{Deg} M=\operatorname{Deg} M / L+l(L)$.

(ii) If $y \in S_{1}$ is sufficiently general and $M$-regular, then $\operatorname{Deg} M \geq \operatorname{Deg} M / y M$.

(iii) If $M$ is a $\mathrm{CM}$ module, then $\operatorname{Deg} M=\operatorname{deg} M$.

The first example of such an extended degree function has been introduced by Vasconcelos. The homological degree of $M$ is defined recursively as

$$
\operatorname{hdeg} M=\operatorname{deg} M+\sum_{i=0}^{d-1}\left(\begin{array}{c}
d-1 \\
i
\end{array}\right) \operatorname{hdeg} \operatorname{Ext}_{S}^{n-i}\left(M, \omega_{S}\right) .
$$

Note that this is well defined because $\operatorname{dim} \operatorname{Ext}_{S}^{n-i}\left(M, \omega_{S}\right) \leq i$ for $i=0, \ldots, n$. In 25] it is shown that hdeg $M$ is indeed an extended degree function.

Another extended degree function was defined by Gunston in his thesis [10, Theorem 3.1.2], the smallest extended degree sdeg. Let us recall its axiomatic description.

Theorem 2.1. There is a unique numerical function sdeg defined on finitely generated graded $S$-modules, satisfying the following conditions:

(i) If $L=H_{\mathfrak{m}}^{0}(M)$, then $\operatorname{sdeg} M=\operatorname{sdeg} M / L+l(L)$.

(ii) If $y \in S_{1}$ is sufficiently general and $M$-regular, then $\operatorname{sdeg} M=\operatorname{sdeg} M / y M$.

(iii) $\operatorname{sdeg}(0)=0$.

We recall important properties of the function sdeg. (See [16] for details.)

(i) sdeg is indeed an extended degree function. For any other extended degree function Deg we have that $\operatorname{sdeg} M \leq \operatorname{Deg} M$ for all finitely generated graded $S$-modules $M$.

(ii) Let $F$ be a finitely generated graded free $S$-module and $U \subset F$ a graded submodule. Then $\operatorname{sdeg} F / U=\operatorname{sdeg} F / \operatorname{gin}(U)$ where $\operatorname{gin}(U)$ is the generic initial submodule of $U$ with respect to the reverse lexicographic term order on $F$.

We briefly recall the construction of $\operatorname{gin}(U)$ because we need this module several times in this paper. For details see, for example, Eisenbud's book [7. Let $e_{1}, \ldots, e_{m}$ be a homogeneous basis for the free graded $S$-module $F$. For a monomial $x^{u} \in S$ we call an element $x^{u} e_{j}$ a monomial in $F$. The (degree) reverse lexicographic term-order $<$ (revlex order for short) is defined as follows: $x^{u} e_{s}<x^{v} e_{t}$ if either $\operatorname{deg} x^{u} e_{s}<\operatorname{deg} x^{v} e_{t}$ or $\operatorname{deg} x^{u} e_{s}=\operatorname{deg} x^{v} e_{t}$ and $x^{u}<x^{v}$ in the usual revlex termorder on $S$ or $\operatorname{deg} x^{u} e_{s}=\operatorname{deg} x^{v} e_{t}, x^{u}=x^{v}$ and $s>t$.

Consider GL $(n)$ as the group of $K$-linear graded automorphisms of $S$ and let $\mathrm{GL}(F)$ be the group of $S$-linear graded automorphisms of $F$. Then $\mathcal{G}=\mathrm{GL}(n) \ltimes$ GL $(F)$ acts on $F$ through $K$-linear graded automorphisms. Recall that a monomial submodule of $F$ is a module generated by monomials of $F$. There exists a non-empty open set $\mathcal{U} \subset \mathcal{G}$ and a unique monomial submodule $U^{\prime} \subset F$ with $U^{\prime}=\operatorname{in}_{>}(g(U))$ for every $g \in \mathcal{U}$ with respect to the revlex order. We call $U^{\prime}$ the generic initial module of $U$ and denote it by $\operatorname{gin}(U)$.

We will also consider the lexicographic submodule $U^{\text {lex }}$ associated to $U \subset F$. The lexicographic order on $F$ is defined by $x^{u} e_{i}>x^{v} e_{j}$ if either $i<j$ or $i=j$ 
and the first non-zero entry of $u-v$ is positive. A lexicographic submodule is a monomial submodule $V \subset F$ such that, for every $i, V_{i}$ is spanned by the first $\operatorname{dim}_{K} V_{i}$ monomials of $F_{i}$ in the lexicographic order. If $U \subset F$ is any graded submodule, then $U^{\text {lex }}$ is the lexicographic submodule of $F$ such that $\operatorname{dim}_{K} U_{i}=$ $\operatorname{dim}_{K}\left(U^{\text {lex }}\right)_{i}$ for all integers $i$.

Later on we will use the fact that $U^{\text {lex }}$ and $\operatorname{gin}(U)$ are Borel-fixed submodules (cf. [7]).

\section{Sequentially Cohen-Macaulay modules}

In this section we derive formulas for degree functions when they are restricted to the class of sequentially Cohen-Macaulay modules. The methods developed here will be very useful in later sections.

Let us briefly recall the definition and some facts about sequentially CohenMacaulay modules. Let $K$ be a field and let $R$ be a standard graded CohenMacaulay $K$-algebra of dimension $n$ with canonical module $\omega_{R}$. The following definition is due to Stanley [21].

Definition 3.1. Let $M$ be a finitely generated graded $R$-module. The module $M$ is said to be sequentially Cohen-Macaulay (sequentially CM modules for short), if there exists a finite filtration

$$
0=M_{0} \subset M_{1} \subset M_{2} \subset \cdots \subset M_{r}=M
$$

of $M$ by graded submodules of $M$ such that each quotient $M_{i} / M_{i-1}$ is CohenMacaulay and $\operatorname{dim} M_{1} / M_{0}<\operatorname{dim} M_{2} / M_{1}<\cdots<\operatorname{dim} M_{r} / M_{r-1}$.

We recall some results from [11] and [12]:

(i) The filtration (1) of a sequentially CM module is uniquely determined and is called the $C M$-filtration of $M$.

(ii) Setting $d_{i}=\operatorname{dim} M_{i} / M_{i-1}$ we have $d_{i}=\operatorname{dim} M_{i}$ for $i=1, \ldots, r$. Furthermore, $\operatorname{dim} M=d_{r}$ and $\operatorname{depth} M=d_{1}$.

(iii) $M$ is sequentially $\mathrm{CM}$ if and only if for all $i=0, \ldots, \operatorname{dim} M$ we have that the modules $\operatorname{Ext}_{R}^{n-i}\left(M, \omega_{R}\right)$ are either 0 or $\mathrm{CM}$ of dimension $i$. In this case,

$$
\begin{aligned}
\operatorname{Ext}_{R}^{n-d_{i}}\left(M, \omega_{R}\right) & \cong \operatorname{Ext}_{R}^{n-d_{i}}\left(M_{i} / M_{i-1}, \omega_{R}\right) \text { for } i=1, \ldots, r \text { and } \\
\operatorname{Ext}_{R}^{n-i}\left(M, \omega_{R}\right) & =0 \text { for } i \neq\left\{d_{1}, \ldots, d_{r}\right\} .
\end{aligned}
$$

(iv) A finite direct sum of sequentially $\mathrm{CM}$ modules is sequentially CM.

(v) $M$ is sequentially $\mathrm{CM}$ if and only if $M / H_{\mathfrak{m}}^{0}(M)$ is sequentially CM.

(vi) Let $y \in R_{1}$ be an $M$-regular element that is also regular on all $\operatorname{Ext}_{R}^{i}\left(M, \omega_{R}\right)$. Then $M$ is sequentially CM if and only if $M / y M$ is sequentially CM.

From now on all modules are assumed to be finitely generated graded modules over $S=K\left[x_{1}, \ldots, x_{n}\right]$.

Sequentially Cohen-Macaulay modules occur frequently. We set

$$
U: I^{\infty}:=\bigcup_{k \geq 0} U:_{F} I^{k}
$$

if $U$ is a submodule of the free $S$-module $F$ and $I \subset S$ is an ideal. Then we have the following remark. 
Remark 3.2. Let $I \subset S$ be a graded ideal. Recall the following definition from [12. The ideal $I$ is said to be of Borel type, if we have for $i=1, \ldots, n$ that

$$
I: x_{i}^{\infty}=I:\left(x_{1}, \ldots, x_{i}\right)^{\infty} .
$$

A Borel-fixed ideal is of Borel-type (see [7, Proposition 15.24]), hence so is the generic initial ideal gin $(I)$ with respect to the reverse lexicographic term order of I. In [12] Herzog, Popescu and Vladoiu proved that if $I$ is a monomial ideal of Borel-type, then $R=S / I$ is sequentially CM.

Observe that the last result is no longer true if $I$ is not a monomial ideal as the following example shows.

Example 3.3. Consider the ideal

$$
I=\left(x_{1}^{2}, x_{1} x_{2}, x_{2}^{2}, x_{1} x_{3}+x_{2} x_{4}\right) \subset K\left[x_{1}, x_{2}, x_{3}, x_{4}\right] .
$$

It defines a double line in $\mathbb{P}^{3}$. Let $R=K\left[x_{1}, x_{2}, x_{3}, x_{4}\right] / I$. Then we have (cf., e.g., [17) that $\operatorname{dim} R=2$ and $\operatorname{Ext}_{S}^{n-1}\left(R, \omega_{S}\right) \cong K$. Hence $R$ is not sequentially CM, but it is of Borel type because

$$
\begin{aligned}
& I: x_{4}^{\infty}=I:\left(x_{1}, x_{2}, x_{3}, x_{4}\right)^{\infty}=I, \\
& I: x_{3}^{\infty}=I:\left(x_{1}, x_{2}, x_{3}\right)^{\infty}=I, \\
& I: x_{2}^{\infty}=I:\left(x_{1}, x_{2}\right)^{\infty}=K\left[x_{1}, x_{2}, x_{3}, x_{4}\right], \\
& I: x_{1}^{\infty}=K\left[x_{1}, x_{2}, x_{3}, x_{4}\right] .
\end{aligned}
$$

The notion of monomial ideals of Borel-type can easily be generalized to modules. Let $F$ be a finitely generated free graded $S$-module with homogeneous basis $e_{1}, \ldots, e_{m}$ and let $U \subseteq F$ be a graded submodule. The module $U$ is said to be of Borel-type if

$$
U: x_{i}^{\infty}=U:\left(x_{1}, \ldots, x_{i}\right)^{\infty} \text { for } i=1, \ldots, n .
$$

As for ideals, we have:

Lemma 3.4. If $U \subset F$ is monomial and of Borel-type, then $F / U$ is sequentially $C M$.

In particular, $F / U$ is sequentially $C M$ if $U=\operatorname{gin}(V)$ or $U=W^{\text {lex }}$ for graded submodules $V, W \subset F$.

Proof. By assumption, we can write $U=\bigoplus_{i=1}^{m} I_{j} e_{j}$ for monomial ideals $I_{j} \subset S$ of Borel-type. Since, by [12, $S / I_{j}$ is sequentially $\mathrm{CM}$ for $j=1, \ldots, m$, we have that $F / U \cong \bigoplus_{j=1}^{m} S / I_{j}$ is sequentially CM because a direct sum of sequentially $\mathrm{CM}$ modules is sequentially $\mathrm{CM}$.

Now, our goal is to show that in the case of sequentially CM modules it is possible to give formulas for several degree functions in terms of certain extension modules. At first we compute the homological degree of a module.

Theorem 3.5. Let $M$ be a sequentially $C M S$-module of dimension d. Then we have

$$
\text { hdeg } M=\operatorname{deg} M+\sum_{i=0}^{d-1}\left(\begin{array}{c}
d-1 \\
i
\end{array}\right) \operatorname{deg} \operatorname{Ext}_{S}^{n-i}\left(M, \omega_{S}\right) .
$$

Moreover, if $M=F / U$ is a presentation of $M$ where $F$ is a finitely generated graded free $S$-module and $U \subset F$ is a graded submodule, then

$$
\text { hdeg } F / U=\operatorname{hdeg} F / \operatorname{gin}(U) \text {. }
$$


Proof. By the definition of the homological degree we have that

$$
\operatorname{hdeg} M=\operatorname{deg} M+\sum_{i=0}^{d-1}\left(\begin{array}{c}
d-1 \\
i
\end{array}\right) \operatorname{hdeg} \operatorname{Ext}_{S}^{n-i}\left(M, \omega_{S}\right) .
$$

Since hdeg $M=\operatorname{deg} M$ for every CM module, the first claim follows.

If $M=F / U$, then

$$
\operatorname{hdeg} M=\operatorname{deg} F / U+\sum_{i=0}^{d-1}\left(\begin{array}{c}
d-1 \\
i
\end{array}\right) \operatorname{deg} \operatorname{Ext}_{S}^{n-i}\left(F / U, \omega_{S}\right) .
$$

By Theorem 3.1 in [1] the Hilbert functions of the graded modules $\operatorname{Ext}_{S}^{n-i}\left(F / U, \omega_{S}\right)$ and $\operatorname{Ext}_{S}^{n-i}\left(F / \operatorname{gin}(U), \omega_{S}\right)$ coincide for all $i$. In particular, these modules have the same degree. Since $F / \operatorname{gin}(U)$ is sequentially CM by 3.4, this proves the second assertion.

We will see in Section 5 that the statement is not true for an arbitrary $S$-module.

For a first application of the theorem we need the following result.

Lemma 3.6. Let $M$ be a finitely generated graded $S$-module. If $y \in S_{1}$ is $M$ regular and $\operatorname{Ext}_{S}^{i}\left(M, \omega_{S}\right)$-regular for all $i$, then the module $\operatorname{Ext}_{S}^{i}\left(M, \omega_{S}\right)$ is $C M$ of dimension $j$ if and only if $\operatorname{Ext}_{S}^{i+1}\left(M / y M, \omega_{S}\right)$ is $C M$ of dimension $j-1$. In this case we have $\operatorname{deg} \operatorname{Ext}_{S}^{i}\left(M, \omega_{S}\right)=\operatorname{deg} \operatorname{Ext}_{S}^{i+1}\left(M / y M, \omega_{S}\right)$.

Proof. The long exact sequence derived from the short exact sequence

$$
0 \rightarrow M(-1) \rightarrow M \rightarrow M / y M \rightarrow 0
$$

splits into short exact sequences

$$
0 \rightarrow \operatorname{Ext}_{S}^{i}\left(M, \omega_{S}\right) \rightarrow \operatorname{Ext}_{S}^{i}\left(M, \omega_{S}\right)(+1) \rightarrow \operatorname{Ext}_{S}^{i+1}\left(M / y M, \omega_{S}\right) \rightarrow 0
$$

from which the assertion follows.

In [24, Conjecture 9.4.1] Vasconcelos conjectured that for every $M$-regular element $y \in S$ we have that hdeg $M \geq \operatorname{hdeg} M / y M$. If $M$ is sequentially $\mathrm{CM}$, then this is true and moreover we can compute the difference hdeg $M-\operatorname{hdeg} M / y M$.

Corollary 3.7. Let $M$ be a sequentially $C M S$-module of dimension d. If $y \in S_{1}$ is $M$-regular, then

$$
\operatorname{hdeg} M=\operatorname{hdeg} M / y M+\sum_{i=1}^{d-2}\left(\begin{array}{c}
d-2 \\
i
\end{array}\right) \operatorname{deg} \operatorname{Ext}_{S}^{n-i}\left(M, \omega_{S}\right) \geq \operatorname{hdeg} M / y M .
$$

In particular, hdeg $M=\operatorname{hdeg} M / y M$ if $\operatorname{dim} M \leq 2$.

Proof. It follows from the local duality theorem, that a prime ideal $P$ of height $i$ is associated to $M$ if and only if $\operatorname{Ext}_{S}^{i}\left(M, \omega_{S}\right)_{P} \neq 0$. Since $M$ is sequentially CM and therefore $\operatorname{Ext}_{S}^{i}\left(M, \omega_{S}\right)$ is zero or CM of dimension $n-i$, the associated prime ideals of $\operatorname{Ext}_{S}^{i}\left(M, \omega_{S}\right)$ are exactly the associated prime ideals of $M$ of height $i$. We deduce that $y$ is also an $\operatorname{Ext}_{S}^{i}\left(M, \omega_{S}\right)$-regular element for all $i$. The long exact Ext-sequence derived from the short exact sequence

$$
0 \rightarrow M(-1) \stackrel{y}{\rightarrow} M \rightarrow M / y M \rightarrow 0
$$


provides, for all $i<n$, short exact sequences of the form

$$
0 \rightarrow \operatorname{Ext}_{S}^{n-i}\left(M, \omega_{S}\right) \stackrel{y}{\rightarrow} \operatorname{Ext}_{S}^{n-i}\left(M, \omega_{S}\right)(+1) \rightarrow \operatorname{Ext}_{S}^{n-i+1}\left(M / y M, \omega_{S}\right) \rightarrow 0 .
$$

Note that $\operatorname{Ext}_{S}^{n}\left(M, \omega_{S}\right)=0$ because $\operatorname{depth} M>0$.

Now, we show the claim by induction on $d$. If $d=1$, there is nothing to prove. Let $d \geq 2$. Observe that $\operatorname{deg} M=\operatorname{deg} M / y M$ and $\operatorname{dim} M / y M=d-1$. It follows that

$$
\begin{aligned}
\operatorname{hdeg} M & =\operatorname{deg} M+\sum_{i=1}^{d-1}\left(\begin{array}{c}
d-1 \\
i
\end{array}\right) \operatorname{deg} \operatorname{Ext}_{S}^{n-i}\left(M, \omega_{S}\right) \\
& =\operatorname{deg} M / y M+\sum_{i=1}^{d-1}\left(\begin{array}{c}
d-1 \\
i
\end{array}\right) \operatorname{deg} \operatorname{Ext}_{S}^{n-i+1}\left(M / y M, \omega_{S}\right) \\
& =\operatorname{deg} M / y M+\sum_{i=1}^{d-1}\left(\left(\begin{array}{c}
d-2 \\
i
\end{array}\right)+\left(\begin{array}{c}
d-2 \\
i-1
\end{array}\right)\right) \operatorname{deg} \operatorname{Ext}_{S}^{n-i+1}\left(M / y M, \omega_{S}\right) \\
& =\operatorname{hdeg} M / y M+\sum_{i=1}^{d-1}\left(\begin{array}{c}
d-2 \\
i
\end{array}\right) \operatorname{deg} \operatorname{Ext}_{S}^{n-i+1}\left(M / y M, \omega_{S}\right) \\
& =\operatorname{hdeg} M / y M+\sum_{i=1}^{d-2}\left(\begin{array}{c}
d-2 \\
i
\end{array}\right) \operatorname{deg} \operatorname{Ext}_{S}^{n-i}\left(M, \omega_{S}\right),
\end{aligned}
$$

which is the desired formula.

Next, we consider the smallest extended degree of a sequentially CM module. To this end we recall some well-known results. For the convenience of the reader we reproduce the short proofs.

Lemma 3.8. Let $M$ be a finitely generated graded $S$-module and $d=\operatorname{dim} M$. Then $\operatorname{deg} M=\operatorname{deg} \operatorname{Ext}_{S}^{n-d}\left(M, \omega_{S}\right)$.

Proof. Denote by $P_{M}$ and $H_{M}$ the Hilbert polynomial and the Hilbert function of $M$, respectively. There is the following formula of Serre (cf., e.g., [3, Theorem 4.4.3]):

$$
H_{M}(j)-P_{M}(j)=\sum_{i=0}^{d}(-1)^{i} \operatorname{dim}_{K} H_{\mathfrak{m}}^{i}(M)_{j}=\sum_{i=0}^{d}(-1)^{i} \operatorname{dim}_{K} \operatorname{Ext}_{S}^{n-i}\left(M, \omega_{S}\right)_{-j} .
$$

Since $\operatorname{dim} \operatorname{Ext}_{S}^{n-i}\left(M, \omega_{S}\right) \leq i$ and $\operatorname{dim} \operatorname{Ext}_{S}^{n-d}\left(M, \omega_{S}\right)=d$, the claim follows by considering $P_{M}$ and the Hilbert polynomial of $\operatorname{Ext}_{S}^{n-d}\left(M, \omega_{S}\right)$ for integers $j \ll$ 0 .

Lemma 3.9. Let $M$ be a finitely generated graded $S$-module and $L=H_{\mathfrak{m}}^{0}(M)$. Then

$$
\operatorname{deg} \operatorname{Ext}_{S}^{i}\left(M / L, \omega_{S}\right)= \begin{cases}\operatorname{deg} \operatorname{Ext}_{S}^{i}\left(M, \omega_{S}\right) & \text { for } i<n, \\ 0 & \text { for } i=n .\end{cases}
$$

Proof. The long exact Ext-sequence derived from the short exact sequence

$$
0 \rightarrow L \rightarrow M \rightarrow M / L \rightarrow 0
$$

and the fact that $\operatorname{Ext}_{S}^{i}\left(L, \omega_{S}\right)=0$ for $i \neq n$ and $l\left(\operatorname{Ext}_{S}^{n}\left(L, \omega_{S}\right)\right)=l(L)$ imply the assertion. 
Now, we are ready for the computation of the smallest extended degree.

Theorem 3.10. Let $M$ be a sequentially $C M S$-module of dimension d. Then we have

$$
\operatorname{sdeg} M=\sum_{i=0}^{d} \operatorname{deg} \operatorname{Ext}_{S}^{n-i}\left(M, \omega_{S}\right)=\operatorname{deg} M+\sum_{i=0}^{d-1} \operatorname{deg}_{\operatorname{Ext}_{S}^{n-i}\left(M, \omega_{S}\right) .}
$$

Proof. Lemma 3.8 yields the second equality. We show the first equality by induction on $d$. If $d=0$, then $M$ is CM and we have that $\operatorname{sdeg} M=\operatorname{deg} M=$ $\operatorname{deg} \operatorname{Ext}_{S}^{n-d}\left(M, \omega_{S}\right)$ where the last equality follows from 3.8. Assume that $d>0$. We consider two cases.

(i) Assume depth $M>0$. Since $M$ is sequentially CM, we can choose an element $y \in S_{1}$ which is $M$-regular and $\operatorname{Ext}_{S}^{n-i}\left(M, \omega_{S}\right)$-regular for all $i$. It follows that

$$
\begin{gathered}
\operatorname{sdeg} M=\operatorname{sdeg} M / y M=\sum_{i=0}^{d-1} \operatorname{deg} \operatorname{Ext}_{S}^{n-i}\left(M / y M, \omega_{S}\right) \\
=\sum_{i=0}^{d-1} \operatorname{deg} \operatorname{Ext}_{S}^{n-i-1}\left(M, \omega_{S}\right)=\sum_{i=1}^{d} \operatorname{deg} \operatorname{Ext}_{S}^{n-i}\left(M, \omega_{S}\right)=\sum_{i=0}^{d} \operatorname{deg} \operatorname{Ext}_{S}^{n-i}\left(M, \omega_{S}\right)
\end{gathered}
$$

where the second equality follows from the induction hypothesis and the third from Lemma 3.6

(ii) Assume depth $M=0$. One of the properties of the smallest extended degree provides

$$
\operatorname{sdeg} M=\operatorname{sdeg} M / H_{\mathfrak{m}}^{0}(M)+l\left(H_{\mathfrak{m}}^{0}(M)\right) .
$$

Note that $l\left(H_{\mathfrak{m}}^{0}(M)\right)=\operatorname{deg} \operatorname{Ext}_{S}^{n}\left(M, \omega_{S}\right)$ by graded local duality. Applying case (i) to the module $M / H_{\mathfrak{m}}^{0}(M)$ and using Lemma 3.9 we get

$$
\begin{aligned}
\operatorname{sdeg} M & =\operatorname{sdeg} M / H_{\mathfrak{m}}^{0}(M)+l\left(H_{\mathfrak{m}}^{0}(M)\right) \\
& =\sum_{i=1}^{d} \operatorname{deg} \operatorname{Ext}_{S}^{n-i}\left(M / H_{\mathfrak{m}}^{0}(M), \omega_{S}\right)+\operatorname{deg} \operatorname{Ext}_{S}^{n}\left(M, \omega_{S}\right) \\
& =\sum_{i=0}^{d} \operatorname{deg} \operatorname{Ext}_{S}^{n-i}\left(M, \omega_{S}\right) .
\end{aligned}
$$

This completes the proof.

Finally, we consider the arithmetic degree.

Theorem 3.11. Let $M$ be a finitely generated graded $S$-module with $d=\operatorname{dim} M$. If for all $i$ the module $\operatorname{Ext}_{S}^{n-i}\left(M, \omega_{S}\right)$ is zero or $C M$, then

$$
\operatorname{adeg} M=\sum_{i=0}^{d} \operatorname{deg} \operatorname{Ext}_{S}^{n-i}\left(M, \omega_{S}\right)=\operatorname{deg} M+\sum_{i=0}^{d-1} \operatorname{deg} \operatorname{Ext}_{S}^{n-i}\left(M, \omega_{S}\right) .
$$

In particular, this formula is true for every sequentially CM module.

Proof. By [24, Proposition 9.1.2] we know that

$$
\operatorname{adeg} M=\sum_{i=0}^{n} \operatorname{deg} \operatorname{Ext}_{S}^{i}\left(\operatorname{Ext}_{S}^{i}\left(M, \omega_{S}\right), \omega_{S}\right) .
$$


Since $\operatorname{Ext}_{S}^{i}\left(M, \omega_{S}\right)$ is zero or CM, Lemma 3.8 provides

$$
\operatorname{deg} \operatorname{Ext}_{S}^{i}\left(\operatorname{Ext}_{S}^{i}\left(M, \omega_{S}\right), \omega_{S}\right)=\operatorname{deg} \operatorname{Ext}_{S}^{i}\left(M, \omega_{S}\right) .
$$

Using $\operatorname{Ext}_{S}^{i}\left(M, \omega_{S}\right)=0$ for $i<n-d$ and $\operatorname{deg} M=\operatorname{deg} \operatorname{Ext}_{S}^{n-d}\left(M, \omega_{S}\right)$ (by Lemma 3.8), we get the claimed equalities. In order to conclude the proof, we note that a sequentially CM module satisfies the assumption of the theorem.

Theorem 3.5. Theorem 3.10 and Theorem 3.11 imply, in particular, the following result.

Corollary 3.12. Let $M$ be a finitely generated graded $S$-module which is sequentially CM. Then we have

$$
\operatorname{deg} M \leq \operatorname{adeg} M=\operatorname{sdeg} M \leq \operatorname{hdeg} M .
$$

Furthermore,

(i) $\operatorname{deg} M=\operatorname{adeg} M$ if and only if $M$ is Cohen-Macaulay.

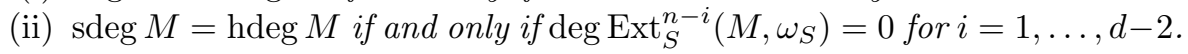

In Section 5 we will see that some of these relations are true in much greater generality.

\section{Buchsbaum modules}

Recall that $S=K\left[x_{1}, \ldots, x_{n}\right]$ is the polynomial ring over the infinite field $K$ with graded maximal ideal $\mathfrak{m}=\left(x_{1}, \ldots, x_{n}\right)$. Let $M$ be a finitely generated graded $S$-module of dimension $d=\operatorname{dim} M$. The module $M$ is called a Buchsbaum module if $d=0$ or $d>0$ and every homogeneous system of parameters $y_{1}, \ldots, y_{d}$ of $M$ is a weak $M$-sequence, i.e.,

$$
\left(y_{1}, \ldots, y_{i-1}\right) M: y_{i}=\left(y_{1}, \ldots, y_{i-1}\right) M: \mathfrak{m} \text { for } i=1, \ldots, d .
$$

We need some properties of Buchsbaum modules. If $M$ is a Buchsbaum module, then:

(i) $\mathfrak{m} H_{\mathfrak{m}}^{i}(M)=0$ for $i=1, \ldots, d-1$.

(ii) If $y_{1}, \ldots, y_{r}$ is part of a homogeneous system of parameters of $M$, then also $M /\left(y_{1}, \ldots, y_{r}\right) M$ is a Buchsbaum module of dimension $d-r$.

Note that property (i) implies that $l\left(H_{\mathfrak{m}}^{i}(M)\right)<\infty$. Thus, local duality provides that $l\left(H_{\mathfrak{m}}^{i}(M)\right)=l\left(\operatorname{Ext}_{S}^{n-i}\left(M, \omega_{S}\right)\right)$ and $\mathfrak{m E x t} \operatorname{Ex}_{S}^{n-i}\left(M, \omega_{S}\right)=0$. An arbitrary module $M$ that only satisfies property (i) is called a quasi-Buchsbaum module. For more details on the theory of Buchsbaum modules we refer to the book of Stückrad and Vogel [22].

In this section we study the behavior of degree functions when they are applied to Buchsbaum modules. In the case of the homological degree, the following result was already noted in [24, Theorem 9.4.1]. It follows immediately from the definition of hdeg because every module $M$ of finite length satisfies hdeg $M=l(M)$.

Proposition 4.1. Let $M$ be a finitely generated graded $S$-module and $d=\operatorname{dim} M$ such that $l\left(\operatorname{Ext}_{S}^{n-i}\left(M, \omega_{S}\right)\right)<\infty$ for $i=0, \ldots, d-1$. Then

$$
\operatorname{hdeg} M=\operatorname{deg} M+\sum_{i=0}^{d-1}\left(\begin{array}{c}
d-1 \\
i
\end{array}\right) l\left(\operatorname{Ext}_{S}^{n-i}\left(M, \omega_{S}\right)\right) .
$$

In particular, this formula is true for every Buchsbaum module. 
Next we compute the smallest extended degree of a Buchsbaum module. The theorem below was first stated in Gunston's thesis [10, Proposition 3.2.3], but with the weaker hypothesis that $M$ is quasi-Buchsbaum. However, Gunston's proof does not work in this generality, because if $M$ is a quasi-Buchsbaum module and $y$ is a homogeneous parameter element for $M$, then $M / y M$ is in general not a quasiBuchsbaum module. For an example of such a module see [14, Example 7.4]. Since Gunston's result is not published elsewhere, we give a proof of the theorem.

Theorem 4.2. Let $M$ be a graded Buchsbaum $S$-module of dimension d. Then we have

$$
\operatorname{sdeg} M=\operatorname{deg} M+\sum_{i=0}^{d-1}\left(\begin{array}{c}
d-1 \\
i
\end{array}\right) l\left(\operatorname{Ext}_{S}^{n-i}\left(M, \omega_{S}\right)\right)=\operatorname{hdeg} M .
$$

Proof. We use induction on $d$. If $d=0$, then $M$ is CM and we have that $\operatorname{sdeg} M=$ hdeg $M=\operatorname{deg} M$. Let $d>0$. Assume that $\operatorname{depth} M=0$ and that the assertion is already shown for modules with positive depth. Then, since $M / H_{\mathfrak{m}}^{0}(M)$ is Buchsbaum, also, we get that

$$
\begin{aligned}
\operatorname{sdeg} & M=\operatorname{sdeg} M / H_{\mathfrak{m}}^{0}(M)+l\left(H_{\mathfrak{m}}^{0}(M)\right) \\
& =\operatorname{deg} M / H_{\mathfrak{m}}^{0}(M)+\sum_{i=1}^{d-1}\left(\begin{array}{c}
d-1 \\
i
\end{array}\right) l\left(\operatorname{Ext}_{S}^{n-i}\left(M / H_{\mathfrak{m}}^{0}(M), \omega_{S}\right)\right)+l\left(\operatorname{Ext}_{S}^{n}\left(M, \omega_{S}\right)\right) \\
& =\operatorname{deg} M+\sum_{i=0}^{d-1}\left(\begin{array}{c}
d-1 \\
i
\end{array}\right) l\left(\operatorname{Ext}_{S}^{n-i}\left(M, \omega_{S}\right)\right) .
\end{aligned}
$$

Here the first equality follows from the properties of sdeg, the second equality since $\operatorname{depth} M / H_{\mathfrak{m}}^{0}(M)>0$ (observe that $l\left(\operatorname{Ext}_{S}^{n}\left(M / H_{\mathfrak{m}}^{0}(M), \omega_{S}\right)=0\right.$ ), and the third one from Lemma 3.9 .

It remains to consider the case depth $M>0$. Choose an $M$-regular element $y \in S_{1}$ and consider the short exact sequence

$$
0 \rightarrow M(-1) \stackrel{y}{\rightarrow} M \rightarrow M / y M \rightarrow 0 .
$$

Observe that $y \cdot \operatorname{Ext}_{S}^{n-i}\left(M, \omega_{S}\right)=0$. Hence the associated long exact Ext-sequence splits into short exact sequences of the form

$$
0 \rightarrow \operatorname{Ext}_{S}^{n-i-1}\left(M, \omega_{S}\right)(+1) \rightarrow \operatorname{Ext}_{S}^{n-i}\left(M / y M, \omega_{S}\right) \rightarrow \operatorname{Ext}_{S}^{n-i}\left(M, \omega_{S}\right) \rightarrow 0 .
$$

Thus, we get that $l\left(\operatorname{Ext}_{S}^{n-i}\left(M / y M, \omega_{S}\right)\right)=l\left(\operatorname{Ext}_{S}^{n-i-1}\left(M, \omega_{S}\right)\right)+l\left(\operatorname{Ext}_{S}^{n-i}\left(M, \omega_{S}\right)\right)$. Since $M / y M$ is again a Buchsbaum module, we may apply the induction hypothesis to it and obtain

$$
\begin{aligned}
\operatorname{sdeg} M & =\operatorname{sdeg} M / y M \\
& =\operatorname{deg} M / y M+\sum_{i=0}^{d-2}\left(\begin{array}{c}
d-2 \\
i
\end{array}\right) l\left(\operatorname{Ext}_{S}^{n-i}\left(M / y M, \omega_{S}\right)\right) \\
& =\operatorname{deg} M+\sum_{i=0}^{d-2}\left(\begin{array}{c}
d-2 \\
i
\end{array}\right)\left(l\left(\operatorname{Ext}_{S}^{n-i-1}\left(M, \omega_{S}\right)\right)+l\left(\operatorname{Ext}_{S}^{n-i}\left(M, \omega_{S}\right)\right)\right) \\
& =\operatorname{deg} M+\sum_{i=0}^{d-1}\left(\begin{array}{c}
d-1 \\
i
\end{array}\right) l\left(\operatorname{Ext}_{S}^{n-i}\left(M, \omega_{S}\right)\right) .
\end{aligned}
$$

Comparing with 4.1 we see that sdeg $M=\operatorname{hdeg} M$ and this concludes the proof. 
There is also a formula in the case of the arithmetic degree.

Proposition 4.3. Let $M$ be a graded Buchsbaum $S$-module of dimension d. Then we have

$$
\operatorname{adeg} M=\sum_{i=0}^{d} l\left(\operatorname{Ext}_{S}^{n-i}\left(M, \omega_{S}\right)\right)=\operatorname{deg} M+\sum_{i=0}^{d-1} l\left(\operatorname{Ext}_{S}^{n-i}\left(M, \omega_{S}\right)\right) .
$$

Proof. This follows from Theorem 3.11 since all modules $\operatorname{Ext}_{S}^{n-i}\left(M, \omega_{S}\right)$ are zero or $\mathrm{CM}$ of dimension 0 . 3.12 ,

Combining the previous results we get a statement that is similar to Corollary

Corollary 4.4. Let $M$ be a graded Buchsbaum S-module of dimension d. Then we have

$$
\operatorname{deg} M \leq \operatorname{adeg} M \leq \operatorname{sdeg} M=\operatorname{hdeg} M
$$

Furthermore,

(i) $\operatorname{deg} M=\operatorname{adeg} M$ if and only if $M$ is Cohen-Macaulay.

(ii) $\operatorname{adeg} M=\operatorname{sdeg} M$ if and only if $\operatorname{deg}_{\operatorname{Ext}_{S}^{n-i}}^{n-}\left(M, \omega_{S}\right)=0$ for $i=1, \ldots, d-2$.

Buchsbaum modules form another class of modules where we can give an affirmative answer to Conjecture 9.4.1 in [24].

Corollary 4.5. Let $M$ be a graded Buchsbaum $S$-module. If $y \in S_{1}$ is $M$-regular, then

$$
\text { hdeg } M=\operatorname{hdeg} M / y M \text {. }
$$

Proof. We have that hdeg $M=\operatorname{sdeg} M=\operatorname{sdeg} M / y M=\operatorname{hdeg} M / y M$ where we used Corollary 4.4 and the fact that $M / y M$ is again a Buchsbaum module.

\section{BOUndS FOR DEGREE FUnCTIONS}

We apply the results of the last sections to compare degree functions and to study their behavior when passing to certain monomial modules. This leads to various bounds.

The starting point is the following refinement of [24, Proposition 9.4.2].

Theorem 5.1. Let $M$ be a finitely generated graded $S$-module. Then we have

$$
\operatorname{deg} M \leq \operatorname{adeg} M \leq \operatorname{sdeg} M \leq \operatorname{hdeg} M .
$$

Using our previous results we can give a new, more conceptual proof. We need an extension of Theorem 3.2 in 23 to modules.

Lemma 5.2. If $U$ is a finitely generated graded submodule of the free graded $S$ module $F$, then we have

$$
\operatorname{adeg} F / U \leq \operatorname{adeg} F / \operatorname{gin}(U) .
$$

Proof. We again use the formula

$$
\operatorname{adeg} F / U=\sum_{i=0}^{n} \operatorname{deg} \operatorname{Ext}_{S}^{i}\left(\operatorname{Ext}_{S}^{i}\left(F / U, \omega_{S}\right), \omega_{S}\right) .
$$


Observe that $\operatorname{Ext}_{S}^{i}\left(\operatorname{Ext}_{S}^{i}\left(F / U, \omega_{S}\right), \omega_{S}\right) \neq 0$ if and only if $\operatorname{dim}_{\operatorname{Ext}_{S}^{i}}^{i}\left(F / U, \omega_{S}\right)=$ $n-i$. Thus, we get

$$
\operatorname{deg} \operatorname{Ext}_{S}^{i}\left(\operatorname{Ext}_{S}^{i}\left(F / U, \omega_{S}\right), \omega_{S}\right) \leq \operatorname{deg}_{E_{S t}^{i}}^{i}\left(F / U, \omega_{S}\right) \leq \operatorname{deg} \operatorname{Ext}_{S}^{i}\left(F / \operatorname{gin}(U), \omega_{S}\right)
$$

where the first estimate is a consequence of Lemma 3.8 and the second inequality follows from the fact that, by [19], $\operatorname{dim}_{K} \operatorname{Ext}_{S}^{i}\left(F / U, \omega_{S}\right)_{j} \leq \operatorname{dim}_{K} \operatorname{Ext}_{S}^{i}\left(F / \operatorname{gin}(U), \omega_{S}\right)_{j}$ for all integers $i, j$.

Proof of Theorem 5.1. We know that $\operatorname{sdeg} M \leq \operatorname{hdeg} M$ is true by the properties of the smallest extended degree. Choose a presentation $M=F / U$ where $F$ is a finitely generated free graded $S$-module and $U$ is a graded submodule of $F$. Then we get

$$
\begin{gathered}
\operatorname{adeg} M=\operatorname{adeg} F / U \leq \operatorname{adeg} F / \operatorname{gin}(U)=\operatorname{sdeg} F / \operatorname{gin}(U) \\
=\operatorname{sdeg} F / U=\operatorname{sdeg} M \leq \operatorname{hdeg} M .
\end{gathered}
$$

Here the second inequality follows from Lemma 5.2 and the third one from Corollary 3.12 because $F / \operatorname{gin}(U)$ is sequentially $\mathrm{CM}$.

In order to estimate sdeg $M$ and hdeg $M$ it seems natural by now to consider a presentation $M=F / U$ where $F$ is a free module and to compare the degrees of $M$ with the ones of $F / \operatorname{gin}(U)$ and $F / U^{\text {lex }}$. This works well to give a lower bound.

Proposition 5.3. Let $M$ be a finitely generated graded $S$-module. Let $M=F / U$ be a presentation of $M$ where $F$ is a finitely generated graded free $S$-module and $U \subset F$ is a graded submodule. Then we have

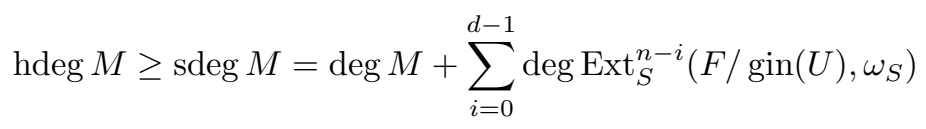

and equality is true if $M$ is a Buchsbaum module.

Proof. We have that

$\operatorname{hdeg} M \geq \operatorname{sdeg} M=\operatorname{sdeg} F / U=\operatorname{sdeg} F / \operatorname{gin}(U)=\sum_{i=0}^{d} \operatorname{deg} \operatorname{Ext}_{S}^{n-i}\left(F / \operatorname{gin}(U), \omega_{S}\right)$.

Here the inequalities and equalities follow from the properties of sdeg (see the remarks after Theorem 2.1) and Theorem 3.10 because $F / \operatorname{gin}(U)$ is sequentially CM by Remark 3.2. If $M$ is a Buchsbaum module, then Corollary 4.4 shows the claimed equality.

In order to give an upper bound for hdeg $M$ we have to restrict ourselves to certain classes of modules because we show in Section 6 that the analogous result is not true for an arbitrary graded $S$-module.

Theorem 5.4. Let $M$ be a finitely generated graded $S$-module which is sequentially $C M$ or a Buchsbaum module. Let $M=F / U$ be a presentation of $M$ where $F$ is a finitely generated graded free $S$-module and $U \subset F$ is a graded submodule. Then we have

$$
\text { hdeg } M \leq \operatorname{hdeg} F / U^{l e x} .
$$


Proof. Consider first the case that $M=F / U$ is sequentially CM. Sbarra proved in his thesis (see [19] for a published proof) that

$$
\operatorname{dim}_{K} \operatorname{Ext}_{S}^{n-i}\left(F / U, \omega_{S}\right)_{j} \leq \operatorname{dim}_{K} \operatorname{Ext}_{S}^{n-i}\left(F / U^{\text {lex }}, \omega_{S}\right)_{j} \text { for all } i, j .
$$

Since $U^{l e x}$ is of Borel-type, it is sequentially CM by Lemma 3.4. Thus, the modules $\operatorname{Ext}_{S}^{n-i}\left(F / U, \omega_{S}\right)$ and $\operatorname{Ext}_{S}^{n-i}\left(F / U^{l e x}, \omega_{S}\right)$ are zero or CM of dimension $i$. Using the inequalities above, this implies that

$$
\operatorname{deg} \operatorname{Ext}_{S}^{n-i}\left(F / U, \omega_{S}\right) \leq \operatorname{deg} \operatorname{Ext}_{S}^{n-i}\left(F / U^{l e x}, \omega_{S}\right) \text { for all } i .
$$

Now Theorem 3.5 shows that hdeg $F / U \leq \operatorname{hdeg} F / U^{l e x}$.

Second, assume that $F / U$ is Buchsbaum. Then we know from Corollary 4.4 that $\operatorname{hdeg} F / U=\operatorname{sdeg} F / U$. Recall that $\operatorname{sdeg} F / U=\operatorname{sdeg} F / \operatorname{gin}(U)$. Applying Theorem 3.10 to $F / \operatorname{gin}(U)$ and $F / \operatorname{gin}(U)^{l e x}=F / U^{\text {lex }}$ and using an argument analogous to the one above in the case of hdeg of sequentially CM modules, we obtain

$$
\operatorname{sdeg} F / \operatorname{gin}(U) \leq \operatorname{sdeg} F / \operatorname{gin}(U)^{l e x}=\operatorname{sdeg} F / U^{l e x} .
$$

It follows that

$$
\operatorname{hdeg} F / U \leq \operatorname{sdeg} F / U^{l e x} \leq \operatorname{hdeg} F / U^{l e x} .
$$

This concludes the proof.

Bounding sdeg is much easier as the proof of Theorem 5.4 shows.

Theorem 5.5. Let $M$ be a finitely generated graded $S$-module. Let $M=F / U$ be a presentation of $M$ where $F$ is a finitely generated graded free $S$-module and $U \subset F$ is a graded submodule. Then

$$
\operatorname{sdeg} M=\operatorname{sdeg} F / \operatorname{gin}(U) \leq \operatorname{sdeg} F / U^{l e x} .
$$

Proof. We know already the first equality. Now the second part of the proof of Theorem 5.4 shows for an arbitrary graded submodule $U \subset F$ that

$$
\operatorname{sdeg} F / \operatorname{gin}(U) \leq \operatorname{sdeg} F / \operatorname{gin}(U)^{l e x}=\operatorname{sdeg} F / U^{l e x} .
$$

Note that formulas for the bounds for hdeg $F / U^{\text {lex }}$ and $\operatorname{sdeg} F / U^{\text {lex }}$ are given by Theorems 3.5 and 3.10. Thus, getting effective estimates amounts to computing degrees of certain extension modules. This can be done efficiently.

Indeed, observe that gin $(U)$ and $U^{\text {lex }}$ are monomial submodules of Borel-type (cf. Lemma 3.4). In Section 7 we will show that one can quickly compute the degree of $\operatorname{Ext}_{S}^{n-i}\left(F / V, \omega_{S}\right)$ where $V \subset F$ is monomial of Borel-type, if one only knows the unique minimal system of monomial generators of $V$. Thus, it is possible to compute our bounds using computer algebra systems such as $\mathrm{CoCoA}$ [4, Macaulay 2 [8] or Singular 9 .

\section{Counterexamples}

The work on this paper started by trying to prove the following conjecture (see Gunston [10, Conjecture 2.5.3] and the book of Vasconcelos [24, page 262]): One of the relations

$$
\operatorname{hdeg} S / I \leq \operatorname{hdeg} S / \operatorname{gin}(I) \text { or hdeg } S / I \geq \operatorname{hdeg} S / \operatorname{gin}(I)
$$

is true for every homogeneous ideal $I \subset S$. 
Very often the lexicographic ideal $I^{\text {lex }}$ associated to $I$ has extremal properties with respect to invariants of $I$. Therefore, it is natural to study the related problem: Is one of the relations

$$
\operatorname{hdeg} S / I \leq \operatorname{hdeg} S / I^{l e x} \text { or } \operatorname{hdeg} S / I \geq \operatorname{hdeg} S / I^{l e x}
$$

true for all ideals I? We have seen that the analogous problem for sdeg has a positive answer because in Theorem 5.5 we proved that

$$
\operatorname{sdeg} S / I=\operatorname{sdeg} S / \operatorname{gin}(I) \leq \operatorname{sdeg} S / I^{l e x} .
$$

Now, we will show that for hdeg all inequalities are false in general. First, we consider the comparison of $I$ and $I^{\text {lex }}$.

Example 6.1. Let $d \geq 3, g<\left(\begin{array}{c}d-2 \\ 2\end{array}\right)$ be integers and set $a:=\left(\begin{array}{c}d-1 \\ 2\end{array}\right)-g$. Consider the ideal

$$
I=\left(x^{2}, x y, y^{d}, y^{d-1} z^{a-d+2}+x t^{a}\right) \subset K[x, y, z, t]=: S .
$$

The ideal $I$ is the homogeneous ideal of an extremal projective curve of degree $d$ and genus $g$ as considered in [15, Example 4.5] (our $a$ is the one in that example plus $d-2$ !). In [15], it is shown that

$$
H_{\mathfrak{m}}^{1}(S / I) \cong K[x, y, z, t] /\left(x, y, z^{a-d+2}, t^{a}\right)(d-1-a),
$$

thus

$$
\operatorname{deg} \operatorname{Ext}_{S}^{3}\left(S / I, \omega_{S}\right)=l\left(H_{\mathfrak{m}}^{1}(S / I)\right)=a(a-d+2) .
$$

Since $\operatorname{dim} S / I=2$, depth $S / I>0$ (for example, the element $t$ is $S / I$-regular), we get

$$
\operatorname{hdeg} S / I=d+a(a-d+2) \text {. }
$$

Next, we compute hdeg $S / I^{\text {lex }}$. The saturation of $I^{\text {lex }}$ is (cf., e.g., [2])

$$
\left(I^{l e x}\right)^{s a t}=\left(x, y^{d+1}, y^{d} z^{a}\right) .
$$

Using, e.g., [12, Corollary 2.6] or [16, Lemma 3.4] we obtain

$$
\operatorname{deg} \operatorname{Ext}_{S}^{3}\left(S / I^{l e x}, \omega_{S}\right)=\operatorname{deg} \operatorname{Ext}_{S}^{3}\left(S /\left(I^{l e x}\right)^{s a t}, \omega_{S}\right)=a .
$$

By considering the Hilbert function of $I$ and using again the theorem of Serre ([3, Theorem 4.4.3]) one gets

$$
\operatorname{deg} \operatorname{Ext}_{S}^{4}\left(S / I^{l e x}, \omega_{S}\right)=l\left(H_{\mathfrak{m}}^{0}\left(S / I^{l e x}\right)\right)=a(d-1) .
$$

It follows that

$$
\operatorname{hdeg} S / I^{l e x}=d+a+a(d-1)=d+a d .
$$

For $d=3, a=2$ we obtain hdeg $S / I=5<9=\operatorname{hdeg} S / I^{\text {lex }}$ and for $d=3, a=5$ we get hdeg $S / I=23>18=\operatorname{hdeg} S / I^{\text {lex }}$. Therefore, in general, there is no relation between hdeg $S / I$ and hdeg $S / I^{l e x}$, not even for two-dimensional rings.

Now, we turn to the comparison of $I$ and $\operatorname{gin}(I)$.

Example 6.2. First, we consider again the ideal $I$ of Example 6.1. Its generic initial ideal has been computed in [15, Proposition 5.5]. It is

$$
\operatorname{gin}(I)=\left(x^{2}, x y, y^{d}, y^{d-1} z^{a-d+2}\right) .
$$

Using [12, Corollary 2.6] we get

$$
\operatorname{deg} \operatorname{Ext}_{S}^{3}\left(S / \operatorname{gin}(I), \omega_{S}\right)=l\left(H_{\mathfrak{m}}^{1}(S / I)\right)=a-d+2 .
$$


Thus, since $\operatorname{depth} S / \operatorname{gin}(I)=1$, we obtain

$$
\operatorname{hdeg} S / \operatorname{gin}(I)=d+a-d+2=a+2 .
$$

Hence, for $d=3$ and $a=2$ we get hdeg $S / I=5>4=\operatorname{hdeg} S / \operatorname{gin}(I)$.

Second, we take a graded Buchsbaum ring $S / J$ of dimension $d \geq 3$ over a polynomial ring $K\left[x_{1}, \ldots, x_{n}\right]$ with $\operatorname{Ext}_{S}^{n-1}\left(S / J, \omega_{S}\right) \neq 0$. Then we have

$$
\operatorname{hdeg} S / J=\operatorname{sdeg} S / J=\operatorname{sdeg} S / \operatorname{gin}(J)<\operatorname{hdeg} S / \operatorname{gin}(J) .
$$

The first equality follows from Corollary 4.4 and the second is a property of sdeg. The third inequality is a consequence of Corollary 3.12, the fact that $S / \operatorname{gin}(J)$ is sequentially CM by Lemma 3.4, and that $\operatorname{Ext}_{S}^{n-1}\left(S / \operatorname{gin}(J), \omega_{S}\right) \neq 0$. The latter we deduce from Sbarra's result in [19], $\operatorname{dim}_{K} \operatorname{Ext}_{S}^{i}\left(S / J, \omega_{S}\right) \leq \operatorname{Ext}_{S}^{i}\left(S / \operatorname{gin}(J), \omega_{S}\right)_{j}$ for all $i, j$.

This shows that, in general, there is no relation between hdeg $S / I$ and hdeg $S / \operatorname{gin}(I)$.

\section{Algorithms}

We have seen that the smallest extended degree has a number of nice properties that are not shared by the homological degree. However, the homological degree has the advantage that is defined by an explicit formula. The goal of this section is to present an algorithm which shows that it is possible to compute effectively the smallest extended degree by using computer algebra systems like CoCoA [4, Macaulay 2 [8], or Singular [9].

The idea for the computation of the smallest extended degree is to use the fact that $\operatorname{sdeg} F / U=\operatorname{sdeg} F / \operatorname{gin}(U)$ for a graded submodule $U$ of a finitely generated graded free $S$-module $F$. This relies on the efficient computation of $\operatorname{deg} \operatorname{Ext}_{S}^{i}\left(F / U, \omega_{S}\right)$ whenever $U$ is of Borel-type. The key result is the following algorithm (see Sturmfels, Trung and Vogel 23] for a related result concerning adeg $S / I$ where $I$ is a monomial ideal).

Algorithm 7.1. Let $U$ be a monomial submodule of a finitely generated graded free $S$-module $F$ with homogeneous basis $e_{1}, \ldots, e_{m}$. Assume that $U$ is a of Boreltype, i.e.,

$$
U: x_{i}^{\infty}=U:\left(x_{1}, \ldots, x_{i}\right)^{\infty} \text { for } i=1, \ldots, n .
$$

Define inductively graded submodules $U_{0}, \ldots, U_{n}$ of $F$ as follows:

(i) Set $U_{0}=M$.

(ii) Assume that $U_{0}, \ldots, U_{i-1}$ are chosen. Put $U_{i}=U_{i-1}: x_{n-i+1}^{\infty}$.

Let $G_{i}$ be the unique minimal system of monomial generators of the monomial module $U_{i}$. Then for all $x^{u} e_{j} \in G_{i}$ we have that $x_{t} \nmid x^{u}$ for $t \geq n-i+1$. Let $V_{i}$ be the monomial submodule of $F$ generated by $G_{i}$ as a $K\left[x_{1}, \ldots, x_{n-i}\right]$-module and denote by $V_{i}^{\text {sat }}$ the saturation of this module as a $K\left[x_{1}, \ldots, x_{n-i}\right]$-module. Then $V_{i}^{\text {sat }} / V_{i}$ is of finite length for $i=0, \ldots, n$ and we have

$$
\operatorname{deg} \operatorname{Ext}_{S}^{n-i}\left(F / U, \omega_{S}\right)=l\left(V_{i}^{s a t} / V_{i}\right),
$$

thus

$$
\operatorname{sdeg} F / U=\sum_{i=0}^{n} l\left(V_{i}^{\text {sat }} / V_{i}\right) .
$$


Proof. It follows from Theorem 3.10 that

$$
\operatorname{sdeg} F / U=\sum_{i=0}^{n} \operatorname{deg} \operatorname{Ext}_{S}^{i}\left(F / U, \omega_{S}\right)
$$

because $F / U$ is sequentially CM by Lemma 3.4 and $\operatorname{Ext}_{S}^{i}\left(F / U, \omega_{S}\right)=0$ for $i<$ $n-\operatorname{dim} F / U$. We have that $U=\bigoplus_{i=1}^{m} I_{j} e_{j}$ for monomial ideals $I_{j} \subset S$ of Boreltype. All computations in Algorithm[7.1 and the Ext-modules commute with finite direct sums. Therefore, we may assume that $F / U=S / I$ for some monomial ideal $I \subset S$ of Borel-type. Now the claim follows from the structure theorem of $\operatorname{Ext}_{S}^{i}\left(S / I, \omega_{S}\right)$ in [12, Corollary 2.6]. Note that in the proofs for Corollary 2.6 in 12, Herzog, Popescu and Vladoiu consider the unique shortest chain of monomial ideals. We changed their computations somewhat. In our notation it can happen that $U_{i}=U_{i-1}$, but then $V_{i}^{\text {sat }}=V_{i}$ and $l\left(V_{i}^{\text {sat }} / V_{i}\right)=0$.

Remark 7.2. Algorithm 7.1 is very fast. If we have computed the unique minimal systems $G_{i}$ of monomial generators of $U_{i}$, then it is easy to determine

$$
U_{i}=U_{i-1}: x_{n-i+1}^{\infty} \text {, }
$$

because we have

$$
G_{i+1}=\left\{x^{u} / x_{n-i+1}^{m_{n-i+1}(u)} e_{j}: x^{u} e_{j} \in G_{i}\right\}
$$

where for a monomial $x_{u}$ we set $m_{t}(u)=\max \left\{s: x_{t}^{s} \mid x^{u}\right\}$. The computation of the length of a quotient of monomial submodules can also very efficiently be done.

Now one can try to extend Algorithm 7.1 to arbitrary modules as follows.

Algorithm 7.3. Let $M$ be a finitely generated graded $S$-module.

(i) Determine a presentation $M=F / U$ where $F$ is a finitely generated graded free $S$-module and $U$ is a graded submodule of $F$.

(ii) Compute $\operatorname{gin}(U)$ with respect to the reverse lexicographic order.

(iii) Find $s:=\operatorname{sdeg} F / \operatorname{gin}(U)$ using 7.1

Then we have that $\operatorname{sdeg} M=s$.

Proof. This follows from the fact that $\operatorname{sdeg} F / U=\operatorname{sdeg} F / \operatorname{gin}(U)$ and $\operatorname{gin}(U)$ is a monomial submodule of $F$ of Borel-type.

Remark 7.4. There is a serious (theoretical) problem with Algorithm 7.3, To compute $\operatorname{gin}(U)$ one usually takes randomly chosen coordinates $y_{1}, \ldots, y_{n}$ of $S$, applies the automorphism $\phi$ of $F$ induced by $x_{i} \mapsto y_{i}$ on $U$, and then computes $\operatorname{gin}(U)$ as the initial module of $\phi(U)$ with respect to the reverse lexicographic term order on $F$. Since for a "generic" $\phi$ we have indeed $\operatorname{gin}(U)=$ in $\phi(U)$, this procedure will almost always correctly determine $\operatorname{gin}(U)$. However, there is no criterion to decide if the monomial module one gets by these computations is in fact the generic initial module of $U$. Hence, one cannot be certain if the result is correct. For practical purposes, the above procedure is of course good enough, since the probability not to get $\operatorname{gin}(U)$ is zero.

In his thesis Gunston [10] has proposed an algorithm for computing sdeg $M$ that uses general hyperplane sections. His procedure also has the theoretical problem that there is no criterium to check if a randomly chosen linear form is indeed general enough. If the module $M$ has dimension $d$, then Gunston's algorithm requires the computation of $d+1$ Gröbner bases, whereas our algorithm has the advantage that it needs just one Gröbner basis computation. 


\section{REFERENCES}

[1] D. Bayer, D. Mumford, What can be computed in algebraic geometry? Computational algebraic geometry and commutative algebra (Cortona, 1991), 1-48, Sympos. Math., Cambridge Univ. Press, Cambridge, 1993. MR 1253986 (95d:13032)

[2] D. Bayer, The division algorithm and the Hilbert scheme, Ph.D. thesis, Harvard University, 1982.

[3] W. Bruns, J. Herzog, Cohen-Macaulay rings, Rev. ed. Cambridge Studies in Advanced Mathematics 39, Cambridge University Press, Cambridge, 1998. MR.1251956 (95h:13020)

[4] CoCoATeam, CoCoA: a system for doing Computations in Commutative Algebra. Available at http://cocoa.dima.unige.it.

[5] N. T. Cuong, L. T. Nhan, Pseudo Cohen-Macaulay and pseudo generalized Cohen-Macaulay modules. J. Algebra 267 (2003), 156-177. MR.1993472 (2004f:13012)

[6] L. R. Doering, T. Gunston, W. V. Vasconcelos, Cohomological degrees and Hilbert functions of graded modules. Amer. J. Math. 120 (1998), no. 3, 493-504. MR1623400 (99h:13019)

[7] D. Eisenbud, Commutative algebra. With a view toward algebraic geometry. Graduate Texts in Mathematics 150, Springer-Verlag, New York, 1995. MR.1322960 (97a:13001)

[8] D. R. Grayson, M. E. Stillman, Macaulay 2, a software system for research in algebraic geometry. Available at http://www.math.uiuc.edu/Macaulay2/.

[9] G. M. Greuel, G. Pfister, H. Schönemann, Singular 2.0 A Computer Algebra System for Polynomial Computations. Centre for Computer Algebra, University of Kaiserslautern (2001). Available at http://www.singular.uni-kl.de.

[10] T. Gunston, Cohomological degrees, Dilworth numbers and linear resolutions. Ph.D. Thesis, Rutgers University, 1998.

[11] J. Herzog, E. Sbarra, Sequentially Cohen-Macaulay modules and local cohomology. Algebra, arithmetic and geometry, Part I, II (Mumbai, 2000), 327-340, Tata Inst. Fund. Res. Stud. Math., 16, Tata Inst. Fund. Res., Bombay, 2002. MR1940671 (2003i:13016)

[12] J. Herzog, D. Popescu, M. Vladoiu, On the Ext-modules of ideals of Borel type. Commutative algebra (Grenoble/Lyon, 2001), 171-186, Contemp. Math. 331, Amer. Math. Soc., Providence, RI, 2003. MR2013165 (2004i:13024)

[13] C. Miyazaki, W. Vogel, Towards a theory of arithmetic degrees. Manuscripta Math. 89 (1996), no. 4, 427-438. MR1383523 (97f:14003)

[14] U. Nagel, Characterization of some projective subschemes by locally free resolutions. Commutative algebra (Grenoble/Lyon, 2001), 235-266, Contemp. Math. 331, Amer. Math. Soc., Providence, RI, 2003. MR2013169 (2004i:14055)

[15] U. Nagel, Non-degenerate curves with maximal Hartshorne-Rao module. Math. Z. 244 (2003), no. 4, 753-773. MR2000458 (2004g:14054)

[16] U. Nagel, Comparing Castelnuovo-Mumford regularity and extended degree: The borderline case. Trans. Amer. Math. Soc. 357 (2005), 3585-3603. MR2146640

[17] U. Nagel, R. Notari, M. L. Spreafico, Curves of degree two and ropes on a line: their ideals and even liaison classes (with R. Notari, M. L. Spreafico). J. Algebra 265 (2003), 772-793. MR.1987029 (2004f:13016)

[18] M. E. Rossi, N. V. Trung, G. Valla, Castelnuovo-Mumford regularity and extended degree. Trans. Amer. Math. Soc. 355 (2003), no. 5, 1773-1786. MR1953524 (2004b:13020)

[19] E. Sbarra, Upper bounds for local cohomology for rings with given Hilbert function. Comm. Algebra 29 (2001), no. 12, 5383-5409. MR.1872238 (2002j:13024)

[20] E. Sbarra, Ideals with maximal local cohomology modules. Rend. Sem. Mat. Univ. Padova 111 (2004), 265-275. MR2076743

[21] R. P. Stanley, Combinatorics and commutative algebra. Second edition. Progress in Mathematics 41, Birkhäuser Boston, Boston, 1996. MR1453579 (98h:05001)

[22] J. Stückrad, W. Vogel, Buchsbaum rings and applications. An interaction between algebra, geometry and topology. Springer-Verlag, Berlin, 1986. MR.0881220(88h:13011a)

[23] B. Sturmfels, N. V. Trung, W. Vogel, Bounds on degrees of projective schemes. Math. Ann. 302 (1995), no. 3, 417-432. MR.1339920 (96i:13029)

[24] W. V. Vasconcelos, Computational methods in commutative algebra and algebraic geometry. Algorithms and Computation in Mathematics 2, Springer-Verlag, Berlin, 1998. MR 1484973 (99c:13048) 
[25] W. V. Vasconcelos, The homological degree of a module. Trans. Amer. Math. Soc. 350 (1998), no. 3, 1167-1179. MR1458335 (98i:13046)

[26] K. Yoshida, A generalization of linear Buchsbaum modules in terms of homological degree. Comm. Algebra 26 (1998), no. 3, 931-945. MR1606198 (99g:13022)

Department of Mathematics, University of Kentucky, Lexington, Kentucky 405060027

E-mail address: uwenagel@ms.uky.edu

FB Mathematik/Informatik, Universität Osnabrück, 49069 Osnabrück, Germany

E-mail address: troemer@mathematik.uni-osnabrueck.de 